\title{
Und Gott war das Wort: Wittgensteins niedrige Absichten
}

\author{
Esther Ramharter, Wien
}

\section{0) Aufriss des Problems}

Wittgenstein insistiert auf einer niedrigen Verwendung von Wörtern:

Während doch die Worte ,Sprache', ,Erfahrung', ,Welt', wenn sie eine Verwendung haben, eine so niedrige Verwendung haben, wie die Worte ,Tisch', ,Lampe', ,Tür'. (PU § 97)

Dieser egalisierenden Wendung scheint jedoch folgende Stelle entgegenzustehen:

Das Leben kann zum Glauben an Gott erziehen [...] - das Leben kann uns diesen Begriff aufzwingen. Er ist dann etwa ähnlich dem Begriff, Gegenstand'. (VB, S. 571)

Es würde uns verwundern, wenn hier statt „Gegenstand“ etwa „Lampe“ stünde (etwas weniger vielleicht „Licht“). Nimmt Wittgenstein also doch eine in irgendeinem Sinn höhere Verwendung eines Begriffs in Anspruch? Man könnte diesem Problem mit dem Hinweis zu entgehen versuchen, dass sich auch das erste Zitat nicht gegen ,höhere Wörter“, sondern nur gegen „höhere Verwendungen“ richtet. Was kennzeichnet aber ein „höheres Wort“", wenn nicht die Verwendung?

Der Begriff „Wort“, den Wittgenstein in Zusammenhängen wie PU $\S 97$ öfters gebraucht, wäre auch hier, als Alternative zu „Gegenstand“, ein naheliegender Kandidat - hat es eine Bedeutung, dass Wittgenstein stattdessen „Gegenstand“" wählt?

${ }^{1}$ Weinberg $(1994,74)$ bezieht sich auch auf diese Stelle, stellt aber - für seine Zwecke gerechtfertigt -,,Gegenstand“" unterschiedslos in eine Reihe mit Wörtern wie ,thoughts“" und ,sensations“. 


\section{1) Der Kontext der zweiten Stelle (VB, S. 571)}

Die Notiz aus dem Jahr 1950, aus der die Stelle stammt, lautet vollständig wiedergegeben:

Ein Gottesbeweis sollte eigentlich etwas sein, wodurch man sich von der Existenz Gottes überzeugen kann. Aber ich denke mir, daß die Gläubigen die solche Beweise lieferten, ihren ,Glauben' mit ihrem Verstand analysieren und begründen wollten, obgleich sie selbst durch solche Beweise nie zum Glauben gekommen wären. ${ }^{2}$ Einen von der ,Existenz Gottes überzeugen' könnte man vielleicht durch eine Art Erziehung, dadurch, daß man sein Leben so und so gestaltet.

Das Leben kann zum , Glauben an Gott' erziehen. Und es sind auch Erfahrungen, die dies tun; aber nicht Visionen, oder sonstige Sinneserfahrungen, die uns die ,Existenz dieses Wesens' zeigen, sondern z.B. Leiden verschiedener Art. Und sie zeigen uns Gott nicht wie ein Sinneseindruck einen Gegenstand, noch lassen sie ihn vermuten. Erfahrungen, Gedanken, - das Leben kann uns diesen Begriff aufzwingen.

Er ist dann etwa ähnlich dem Begriff „Gegenstand”. (VB, S. 571)

Ein Hinweis aus demselben Jahr auf Karl Barth (VB, S. 571) macht deutlich, dass Wittgenstein sich zu dieser Zeit zumindest ein wenig mit Gottesbeweisen beschäftigt hat (auch in den Jahren davor gibt es einige Notizen dazu). Ich behaupte keineswegs, dass Wittgenstein sich eingehender mit einem der „Klassiker“ unter den Gottesbeweisen auseinandergesetzt hat, dennoch möchte ich etwas weiter ausholen und einige Bemerkungen $\mathrm{zu}$ Anselms Gottesbeweis machen, die, so scheint mir, Wittgensteins Überlegung in ein interessantes Licht rücken. Ich versuche zu zeigen, dass es Motivlagen gibt, die Wittgenstein in der Wahl des Wortes „Gegenstand“ bestimmt haben könnten ${ }^{3}$ und die jenen, die Anselm von Canterbury ,id quo maius cogitari non potest" konzipieren haben lassen, sehr ähnlich sind.

Anselms Ontologisches Argument ist jenen von Descartes, Leibniz, etc. vom logischen Standpunkt ${ }^{4}$ überlegen, weil ,id quo maius cogitari non

\footnotetext{
${ }^{2}$ Wittgenstein scheint sich hier Karl Barth in einem eher kontroversen Punkt (vgl. Flasch 1989, 23) anzuschließen, nämlich darin, dass die Erfinder von Gottesbeweisen mit ihren Beweisen nicht überzeugen wollten.

${ }^{3}$ Ein Motiv, das man für etwas hat, muss natürlich keineswegs eine Grundlage einer bewussten Entscheidung sein.

${ }^{4}$ Das bedeutet nicht: hinsichtlich der Überzeugungskraft.
} 
potest“ - wegen der Neutralität des ,maius“ - diejenigen Zuschreibungen, die größer machen, im Unterschied zu den ,pperfectiones“ nicht inhaltlich einschränkt. Größer wird ein Ding für Anselm dadurch, dass ihm eine Eigenschaft mehr zukommt. ${ }^{5}$ „Eigenschaft“ ist ein logischer Begriff, „Perfektion“ nicht (sondern ein metaphysischer). Die folgende formale Darstellung kann sowohl als eine Formalisierung eines Arguments im Sinne Descartes' oder Leibniz' angesehen werden als auch als eine inadäquate Rekonstruktion des Anselmschen Beweises selbst. Die Relevanz der Unterscheidung zwischen rein logischen und metaphysisch-inhaltlichen Begriffen lässt sich daran jedenfalls gut verdeutlichen. Zunächst in Anselmscher Terminologie:

Es soll gezeigt werden:

Rg Gott existiert in Wirklichkeit. ${ }^{6}$

\section{Rekonstruktion mittels Prädikatenlogik 1. Stufe}

Die Prämissen lauten:
$\neg \exists \mathrm{xGxg}$
Es gibt nichts, das größer als Gott ist.
$\forall \mathrm{x}(\neg \mathrm{Rx} \rightarrow \exists \mathrm{yGyx}) \quad \mathrm{Zu}$ allem, was nicht wirklich ist, gibt es etwas Größeres.

Dieses Argument überzeugt nicht, weil (die Variable) G eine inhaltlich (beliebig) bestimmbare Eigenschaft wie R auch ist. Es gibt daher keinen logischen Zusammenhang zwischen „,größer sein“ und „real existieren“. In dieser Formulierung muss also (in der zweiten Prämisse) das implizit vorausgesetzt werden, woraus eigentlich die Überzeugungskraft des Beweises kommen sollte. Wenn Descartes sagt, ,[E]s steht mir nicht frei, Gott ohne Dasein - d.h. das vollkommenste Wesen ohne höchste Vollkommenheit zu denken.“ (Descartes Meditationen, 5. Meditation, S. 60), dann kann man

${ }^{5}$ Er verwendet „Existenz in Realität“ als Eigenschaft, und er gibt keine weitere Einschränkung für das, was größer macht, an.

${ }^{6}$ Die Debatte über „Existenz“ und „,notwendige Existenz“ werde ich hier nicht behandeln. Den Standpunkt von Phillips 1963 und Phillips 1990 finde ich sich sehr berücksichtigenswert. Dort werden auch die Beiträge von Malcolm, Findlay, etc. miteinbezogen. 
dafür (wenn man das Argument als indirekten Beweis führt), die obige Formalisierung übernehmen, wobei G für „,vollkommener“ steht. Dass aber „Existenz“ eine „Vollkommenheit“" ausmacht (daher aus Nicht-Existenz Nicht-Vollkommenheit folgt), ist eben eine inhaltliche Aussage, und keine logische Konsequenz; wenn man dagegen „Existenz“ als Prädikat auffasst, dann ist das Zutreffen dieses Prädikats auf ein Wesen „über das nichts Größeres“ gedacht werden kann, eine logische Folge, das zeigt die folgende Rekonstruktion:

Rekonstruktion mittels Prädikatenlogik 2. Stufe

$\mathrm{Mx}:=\neg \exists \mathrm{F}(\neg \mathrm{Fx})$,worüber nichts Größeres gedacht werden kann" wird definiert

Mg Diese Eigenschaft (2.Stufe) wird Gott zugesprochen. (Es gibt nichts/keine Eigenschaft, das/die Gott fehlt.)

Daraus $\mathrm{Rg}$ zu folgern, ist trivial, aber funktioniert. ${ }^{7}$

Der Beweis funktioniert gerade deswegen, weil einerseits zunächst keine verschieden ,hohen“ Begriffe verwendet werden - wir sprechen über Dinge und über Gott, ob sie existieren, ebenso wie, ob sie blau sind oder wohlschmeckend -, andererseits aber das ,maius“ einen besonderen Stellenwert - eine spezielle Art eines ,hohen“ Gebrauchs - dadurch erhält, dass es als etwas Übergeordnetes Besonderes leistet, nämlich Existenz als logische Konsequenz liefert.

„Worüber es nichts Größeres gibt“" ist (1) nicht selbst eine Eigenschaft wie ein Sinneseindruck, aber (2) durch solche Eigenschaften, die wir sonst zur Charakterisierung von (wahrnehmbaren) Dingen verwenden, definierbar und verwendet (3) alle diese Eigenschaften unterschiedslos, wählt also keine inhaltlich aus (im Unterschied zur zweiten Prämisse in der ersten Rekonstruktion). Schon vor einem eingehenden Vergleich beginnt man hier eine Verwandtschaft mit Wittgensteins „Gegenstand“ zu ahnen.

\footnotetext{
${ }^{7}$ Und es ist die einzige Weise, wie man „maius“ und „Existenz in Wirklichkeit“ im Rahmen einer der üblichen Logiken so fassen kann, dass die gewünschte Folgerung aus rein logischen Gründen - ohne zusätzliches Postulat - gelingt.
} 


\section{2) Der Kontext der ersten Stelle (PU § 97)}

In Bezug auf die erste Stelle gilt es einige begriffliche Klarstellungen zu dem Wortfeld um „niedrig“, „,tief“, ,,hoch“, etc. vorzunehmen. ${ }^{8}$

Wittgenstein mokiert sich über die Neigung der Philosophen, für die Wörter, die sie gebrauchen, eine außerordentliche, reine und erhabene Bedeutung in Anspruch zu nehmen. Aber ist es, auf der anderen Seite, selbstverständlich, dass das Wort „Tisch“ ausschließlich eine niedrige Verwendung hat? Gewiß hat es nicht nur eine einzige Verwendung, denn so mancher geht zum Tisch des Herrn, um das Heilige Abendmahl stehend einzunehmen - weil kein Tisch da ist. Und an einem sonnigen September haben zwei Kontrahenten in einer schwierigen Verhandlung ihre Karten ganz offen auf den Tisch gelegt, während sie im Stadtpark umherschlenderten. Wird in diesen Fällen das Wort „Tisch“ nicht auf eine überhöhte Weise verwendet? Sind das schon erste Anzeichen philosophischer Infektion? (Heinrich 2003, S. 114)

Natürlich gibt es Wörter mit verschieden ,hoher“ Bedeutung (wobei dieses „hoch“ auch in einem höheren Sinn verwendet wird, also nicht im Sinne einer Längenangabe): Es gibt Wörter einerseits für Heiliges, Profanes, Vulgäres, Erhabenes, Niederträchtiges und Edles. Ein Tisch etwa wird geweiht und erhält dadurch eine ,höhere“ Bedeutung.

Nun ist aber der Vorgang, in dem sich solch eine Redeweise einbürgert, von außen gesehen ein ziemlich gewöhnlicher Vorgang, nicht grundsätzlich verschieden von jenem, in dem sich irgendeine gewöhnliche, (im überhöhten Sinn) niedrige Verwendung des Wortes „Tisch“ eingebürgert hat [...]. (Heinrich 2003, S.114f)

Egal, wie „hoch“ Begriffe in ihrer Bedeutung oder Verwendung sind, wie wir sie gebrauchen, lernen wir immer auf die gleiche „niedrige“ Weise. ${ }^{9}$

Werfen wir jetzt einen Blick auf die Stelle, um die es bei meinem Vergleich geht:

Das Denken ist mit einem Nimbus umgeben. - Sein Wesen, die Logik, stellt eine Ordnung dar, und zwar die Ordnung a priori der Welt, d.i. die Ordnung der Möglichkeiten, die Welt und Denken gemeinsam sein muß. Diese Ordnung aber, scheint es, muß höchst einfach sein. Sie ist vor aller Erfahrung; muß sich durch die ganze Erfahrung hindurchziehen; ihr selbst darf keine erfahrungs-

${ }^{8}$ Zum „Höheren“ im Tractatus siehe Donatelli 2005.

${ }^{9}$ Insbesondere lernen wir auch das ,maius“, das Prädikat zweiter Stufe im Gottesbeweis, wie jedes andere Zeichen zu verwenden. 
mäßige Trübe oder Unsicherheit anhaften. - Sie muß vielmehr vom reinsten Kristall sein. Dieser Kristall aber erscheint nicht als eine Abstraktion, sondern als etwas Konkretes, ja als das Konkreteste, gleichsam Härteste. (Log. Phil. Abh. No. 5.5563.)

Wir sind in der Täuschung, das Besondere, Tiefe, das uns Wesentliche unserer Untersuchung liege darin, daß sie das unvergleichliche Wesen der Sprache zu begreifen trachtet. D.i., die Ordnung, die zwischen den Begriffen des Satzes, Wortes, Schließens, der Wahrheit, der Erfahrung, u.s.w. besteht. Diese Ordnung ist eine Über-Ordnung zwischen - sozusagen - Über-Begriffen. Während doch die Worte „Sprache”, „Erfahrung”, „Welt”, wenn sie eine Verwendung haben, eine so niedrige haben müssen, wie die Worte „Tisch”, „Lampe”, „Tür”. (PU §97)

Wenn wir etwas Tiefe oder Höhe zusprechen, besagt das schlicht, dass wir es wichtig nehmen. ${ }^{10}$ „Nimbus“ besagt, dass wir es $z u$ wichtig nehmen. Diese Feststellung ändert aber nichts daran, dass es diesen Stellenwert eben hat. Zum Feuer etwa merkt Wittgenstein an:

Nichts spricht dafür, warum das Feuer mit solchem Nimbus umgeben sein sollte. Und, wie seltsam, was heißt es eigentlich „es schien vom Himmel gekommen zu sein"? von welchem Himmel. Nein, es ist gar nicht selbstverständlich, daß das Feuer so betrachtet wird; aber es wird eben so betrachtet. (BEE, Item 143, S. 10)

Wittgenstein spricht im darauf folgenden Absatz auch von Tiefe, und meint, dass man etwa im Fall von Siegfried und Brunhilde im neueren Nibelungenlied sieht, dass diese vom Zusammenhang mit dem Verbrennen eines Menschen herrührt.

\section{3) Die niedrige Ebene und der gehandhabte Nimbus}

Wittgensteins „Gegenstand“ bezieht ebenso wie Anselms „quo maius cogitari non potest" seine Bedeutung aus Eigenschaften (zur Erklärung, was „Gegenstand“ heißt, müssten wir Beschreibungen heranziehen, es würde nichts fruchten, auf Dinge zu zeigen), und zwar aus als unterschiedslos behandelten Eigenschaften (Gegenstand zu sein schließt a priori keine Eigenschaft - eines Erfahrungsdinges - aus), findet aber auf Individuen Anwen-

${ }^{10}$ Missdeutungen unserer Sprachformen sind so tief, wie uns unsere Sprache wichtig ist, ebenso Witze (PU §111). 
dung $^{11}$ - im Unterschied zu „Wort“ etwa. Das ist die Basis dafür, dass Anselms Argument logisch funktioniert. Dafür, dass auch Wittgenstein in diese Richtung gedacht haben könnte, lassen sich freilich nur Indizien nennen.

Das Wesen Gottes verbürge seine Existenz d.h. eigentlich, daß es sich hier um eine Existenz nicht handelt.

Könnte man denn nicht auch sagen, das Wesen der Farbe verbürge ihre Existenz? Im Gegensatz etwa zum weißen Elephanten. Denn es heißt ja nur: Ich kann nicht erklären, was ,Farbe' ist, was das Wort „Farbe“ bedeutet, außer an Hand der Farbmuster. Es gibt also hier nicht ein Erklären, ,wie es wäre, wenn es Farben gäbe'.

Und man könnte nun sagen: Es läßt sich beschreiben, wie es wäre, wenn es Götter auf dem Olymp gäbe aber nicht: ,wie es wäre, wenn es Gott gäbe'. Und damit wird der Begriff, Gott' näher bestimmt. (VB, S. 566)

Dieses Beispiel zeigt, ${ }^{12}$ dass Wittgenstein auch damit ringt, was aus einem Begriff Gottes folgen kann. Die Stelle aus den Philosophischen Untersuchungen, in der er das Wort „Gegenstand“ wählt, formuliert er wenig später.

Neben dieser Motivation von der Funktion(sweise) her, lassen sich aber auch noch andere Vorzüge des Begriffs „Gegenstand“ gegenüber den nahe liegenden Alternativen angeben: „Gegenstand“ ist

(1) kein religiöser Begriff, in diesem Sinn kein „höherer“ Begriff und nicht mit einem Nimbus umgeben ${ }^{13}$

(2) ein Metabegriff (nicht nur in formaler Hinsicht, sondern auch als Entsprechung der Idee, dass Gott mit den Dingen etwas zu tun haben muss, aber doch ohne eines von ihnen $\mathrm{zu} \operatorname{sein}^{14}$ )

${ }^{11}$ Wittgenstein verwendet, insbesondere $\mathrm{zu}$ der Zeit, in der diese Bemerkungen entstanden sind, bis auf wenige Ausnahmen ,Gegenstand“ im Sinn von „Erfahrungsgegenstand“, nicht in irgendeiner weiteren Bedeutung wie „Thema“ etwa.

12 Interessant wäre hier auch eine Untersuchung der Rolle des monotheistischen Gottesbilds für derlei Überlegungen. Offenkundig funktioniert der Terminus „Gott" nicht nur in politisch-praktischen Kontexten (vgl. die Arbeiten von Jan Assmann), sondern auch im Zusammenhang mit Gottesbeweisen im Monotheismus grundsätzlich anders als bezogen auf viele Götter. Man kann sich etwa kaum vorstellen, dass es für jeden Gott einen spezifischen Gottesbeweis gibt.

13 Jedenfalls nicht in diesem Kontext - beim Nachdenken über Sprache wäre das möglicherweise anders. 
(3) kein rein sprachlicher Begriff (wie etwa „Wort") - in dieser Hinsicht nimmt Wittgenstein die Religion ernst: Im Anfang war nicht das Wort, sondern - für uns - die Dinge des Lebens/das Leben.

(4) ein Begriff, dessen Gebrauch wir in diesem Zusammenhang als nicht metaphorisch bezeichnen würden, als nicht ,höher“ in dem Sinn ${ }^{15}$, der in der Darstellung von Heinrich thematisiert ist. Wittgenstein verwendet z.B. nicht Licht oder Leuchte; er hätte eine der viel konkreteren Metaphern der Bibel verwenden können (für eine sprachanalytische Studie dieser Metaphern siehe Hartl 2008).

Diese Punkte treffen sämtlich auch auf Anselms Konzeption ,id quo maius cogitari non potest" zu. Auch Anselm hält (1) durch die Verwendung des neutralen „maius“ mit einem Nimbus oder Tiefe versehene Begriffe wie Güte, Allmacht, etc. in seinem Gottesbeweis noch fern. Anselms ,quo maius cogitari non potest" kommt (2) wie „Gegenstand" nicht durch (konkrete) sinnlich wahrnehmbare Dinge zu seiner Bedeutung, sondern durch sämtliche irgendwelche Dinge bestimmenden Eigenschaften. Es ist sinnlos, jemand einen Gegenstand hin zu halten und - in der Absicht ihm über diesen Gegenstand etwas mitzuteilen - zu sagen: „Das ist ein Gegenstand“ (in der Hinsicht, in der es auch sinnlos ist zu sagen, ,dieser Stab hat eine Länge“ (vgl. PU §251)). Ob etwas ein Gegenstand ist, stellen wir nicht fest, indem wir hingreifen oder ihn anschauen, aber dennoch ist „Gegenstand" auf (Sinnen-)Dinge anwendbar, also kein auf Sprache allein bezogener Begriff - genau wie „quo maius cogitari potest“16 (3). Mit einem metaphorischen Begriff schließlich hat man es (4) bei Anselm auch nicht zu tun.

Wittgensteins Beschäftigung mit Ontologischen Argumenten ist, wenn auch in ihrem Ausmaß unklar, als Tatsache doch unumstritten. Dass

${ }^{14}$ Vergleiche dazu Phillips 1990, 12.

15 Auch dem metaphorischen Gebrauch stellt Wittgenstein den niedrigen Gebrauch eines „Damit-Leben“ gegenüber: „Wenn Faust sagt ,Wer darf ihn nennen, wer ihn bekennen, etc.', so könnte man ihn fragen: Gebrauchst Du hier nur ein poetisches Gleichnis, oder drängt sich Dir dieser Begriff auf, und lebst Du mit ihm?“ (BEE, Item $174,1 v$ )

${ }^{16}$ Ein Unterschied besteht allerdings darin, dass Gegenstand zu sein auf alle Dinge zutrifft, „quo maius cogitari non potest"“ allerdings - in Anselms Vorstellung - nur auf Eines. 
umgekehrt auch Anselm Überlegungen der Art, wie sie später Wittgenstein anstellt, keineswegs fremd sind, erkennt man bei Beschäftigung mit der Tradition, die Anselm prägt: einer Tradition des Rationalisierens des Glaubens, die sich auf die Grammatik konzentriert und auf einem niedrigen Gebrauch von Wörtern besteht. In der Auseinandersetzung seines Lehrers Lanfrank mit Berengar von Tours steht Anselm auf der Seite Berengars (siehe Flasch 1989, S. 42). Dieser argumentiert im Streit um die Transsubstantiationslehre $^{17}$, dass diese wider die Grammatik wäre. Berengar insistiert darauf, dass das Brot Brot bleiben muss, auch oder gerade wenn es Leib Christi sein soll, ${ }^{18}$ weil sonst die Grammatik Widersprüche hervorbringt.

[Die biblische Satzwahrheit] muss nach den Regeln der significatio ausgelegt werden, die in der Grammatik gelten. Danach sind die angestammten Satzteile in der Lage einen anderen zusätzlichen und höheren Sinn zu erhalten, ohne ihre Grundbedeutung zu verlieren [...]; etwas anderes ist es, daß sie Brot und Wein sind, und etwas anderes, daß sie der Leib und das Blut des Herrn sind [...]. (Hödl 1990, S. 74f)

Das sachgebundene, „niedrige“ Denken gewährleistet, dass Bedeutungen und Wörter sich nicht verselbständigen. Dieses selbe Denken ist es auch, auf das sich Anselm in seinem Gottesbeweis und seinem Disput mit Gaunilo immer beruft. Etwa ist der Gottesbegriff für ihn nicht a priori gegeben (vgl. Flasch 1989, S. 17). Nirgendwo fordert Anselm für die Erkenntnis Gottes irgendeine Art besonderen Vermögens.

Was Anselm das Denken ist, ist Wittgenstein die Sprache. Wittgenstein sagt, das Leben zwingt uns den Begriff „Gott“" auf.

Wie wird uns das Wort, Gott' beigebracht (d.h. sein Gebrauch)? Ich kann davon keine ausführliche systematische Beschreibung geben. Aber ich kann sozusagen Beiträge zu der Beschreibung machen; ich kann darüber manches sagen und vielleicht mit der Zeit eine Art Beispielsammlung anlegen. (VB, S.567)

${ }^{17}$ Wie aus einigen wenigen harmlosen Worten ein die Kirchengeschichten prägender Streit entstehen konnte, sieht man sehr gut in Macy 1999.

${ }^{18}$ Die Verbindung zwischen den beiden Sorten „niedrigen Gebrauchs“ besteht in „primitiven“" Praktiken. Das Wort „Brot“" lernen wir beim Essen. 
Man kann wohl nicht behaupten, dass Wittgenstein hier eine neuartige ${ }^{19}$ Erklärung zum Gebrauch von Wörtern geben würde, bemerkenswert ist viel mehr, dass er dieselbe Erklärung gibt wie sonst: auch „Gott" lernen wir „niedrig“ gebrauchen, in gewöhnlichen Vorgängen, anhand von Beispielen. Nichts unterscheidet diesbezüglich (wie etwa auch beim „Tisch des Herrn“ in Heinrichs Beispiel) den Begriff „Gott" von irgendeinem anderen Begriff. Auch Anselm besteht darauf, dass sein „,(id) quo maius cogitari non potest" auf der niedrigen Ebene bleibt. Anselm und Wittgenstein aber haben verschiedene Gegner: Anselm muss Gott davor bewahren, nur jenseits des Rational-Erkennbaren zu sein (ob Anselm hier Gott vor Anderen oder vor Anselm schützen muss, wird gestritten), Wittgenstein muss den Nimbus abwehren, der Gott ungerechtfertigt erhöhen könnte, und er erinnert daran, dass wir das Wort „Gott“ lernen wie alle anderen Wörter „niedrig“.

${ }^{19}$ Im Vergleich zu seinen früheren Erklärungen 


\section{Literatur}

Descartes, René 1993: Meditationen über die Grundlagen der Philosophie (Meditationen), Hamburg: Felix Meiner Verlag.

Donatelli, Piergiorgio 2005: “The Problem of 'The Higher' in Wittgenstein's Tractatus". In: Phillips / Von der Ruhr 2005, 11-38.

Flasch, Kurt 1989: Einleitung zu Kann Gottes Nicht-Sein gedacht werden? Die Kontroverse zwischen Anselm von Canterbury und Gaunilo von Marmoutiers, Mainz: Dieterich'sche Verlagsbuchhandlung.

Hartl, Johannes 2008: Metaphorische Theologie. Grammatik, Pragmatik und Wahrheitsgehalt religiöser Sprache. Berlin: Lit Verlag.

Hödl, Ludwig 1990: „Die theologischen Auseinandersetzungen mit Berengar von Tours im frühscholastischen Eucharistietraktat De corpore Domini“". In: Ganz, Peter / Huygens, R. B. C. / Niewöhner, Friedrich (Hg.) Auctoritas und Ratio. Studien zu Berengar von Tours: Wiesbaden: Harrassowitz, 69-88.

Heinrich, Richard 2003: „Tisch, Lampe, Tür”. In: Verzauberung, Methode und Gewohnheit. Skizzen zur philosophischen Intelligenz. Wien: Edition Roesner.

Macy, Gary 1999: Treasures from the Storeroom. Medieval Religion and Eucharist. Collegeville: The Liturgical Press.

Phillips, Dewi Zaphaniah 1963: „Philosophy, Theology and the Reality of God”. The Philosophical Quarterly 3. Zitiert nach: Phillips, Dewi Zaphaniah 1993: Wittgenstein and Religion. New York: St. Martin's Press, 1-9.

Phillips, Dewi Zaphaniah 1990: „Sublime Existence”, Archivio Di Filosofia LVIII. Zitiert nach: Wittgenstein and Religion. New York: St. Martin's Press, 10-22.

Phillips, Dewi Zaphaniah / Von der Ruhr, Mario (Hg.) 2005: Religion and Wittgenstein's Legacy. Aldershot / Burlington: Ashgate.

Weinberg, Jonathan 1994: „Picturing God: Wittgenstein on Religion, Science and Superstition“. The Harvard Review of Philosophy 4(1), 64-75.

Wittgenstein, Ludwig 1998-2000: Wittgenstein's Nachlass: The Bergen Electronic Edition. Oxford: Oxford University Press. (BEE)

Wittgenstein, Ludwig 1984: Philosophische Untersuchungen. In: Tractatus logicophilosophicus, Tagebücher 1914-1916, Philosophische Untersuchungen, Suhrkamp Werkausgabe, Bd. 1. Frankfurt am Main: Suhrkamp. (PU)

Wittgenstein, Ludwig 1999 Vermischte Bemerkungen. In: Über Gewißheit, Suhrkamp, Werkausgabe, Bd. 8. Frankfurt am Main: Suhrkamp. (VB) 
